\title{
Characteristics of Developmental Differences between Fertile and Aborted Ovules in Camellia oleifera
}

\author{
Chao Gao \\ Institute for Forest Resources \& Environment of Guizhou, Guizhou University, Guiyang 550025, \\ China \\ Rui Yang \\ College of Forestry, Guizhou University, Guiyang 550025, China \\ Deyi Yuan ${ }^{1}$ \\ Key Laboratory of Cultivation and Protection for Non-Wood Forest Trees of Ministry of Education \\ and the Key Laboratory of Non-Wood Forest Products of Forestry Ministry, Central South University \\ of Forestry and Technology, Changsha 410004, China
}

\begin{abstract}
Additional INDEX words. abortion, embryo sac, embryology, plant reproductive biology, seed
Abstract. Camellia oleifera is an important woody edible oil plant in southern China. In this study, the developmental differences in ovules at different positions in the ovary of $C$. oleifera were observed. The developmental type and characteristics of aborted ovules, ratios of normal and aborted ovules, and their developmental differences after flowering were examined. Ovules near the stylar end and in the middle exhibit normal development and are able to form embryo sacs; lower ovules near the pedicel end are usually aborted. The proportion of abortion of four examined cultivars ranges from $\mathbf{1 0 . 2 \%}$ to $\mathbf{3 3 . 3 \%}$. Aborted ovules can be divided into four categories: 1 ) nascent egg apparatus lacking distinguishable cells; 2) completely absent egg apparatus structure consisting of flocculent tissue; 3) lack of tissue, comprising only integument cells; and 4) the inner integument not constituting a micropyle channel, with incomplete egg apparatus development and generating abnormal ovules. At 120 days after pollination (DAP), significant distinguishable size differences were found between fertile and aborted ovules; aborted ovules ceased growth at 180 DAP. On fruit maturation, aborted seeds were still attached to the placenta.
\end{abstract}

In angiosperms, the ovule is the precursor of the seed and the site of embryo sac development. In turn, female reproductive unit development, double fertilization, and embryo and endosperm growth occur in the embryo sac. Thus, the ovule has an important function in the reproductive system and life cycle of angiosperms (Hu, 2005; Shi and Yang, 2011; Skinner et al., 2004). Ovules are usually buried deep in the ovary, positioned on the placenta in different patterns. Before fertilization, the ovule contains basic parts, including the nucellus, integument, and micropyle, and is connected to the placenta via the funiculus $(\mathrm{Hu}, 2005)$. Formation of a mature and fertile ovule begins with ovule primordial division, with the primordial front end developing into the nucellus and the base into the funiculus. A ring-shaped protrusion developing into the integument emerges within the vicinity of the base of the nucellus. Ovules typically have two layers of integument, with the inner integument appearing first and the outer integument forming thereafter. The integument grows upward to surround the nucellus and forms an apical micropyle (Endress, 2011). The embryo sac arises in the nucellus concurrent with ovule development. Female gametophyte production is divided into two stages: megasporogenesis and female gametophyte

Received for publication 10 May 2017. Accepted for publication 16 June 2017. This work was supported by the National Natural Science Foundation of China (31170639), the Science and Technology Planning Projects of Guizhou (Qian Ke He JZ[2014]200211) and the Major Project of Guizhou Province (Qian Ke He Major Project [20163022-06]).

${ }^{1}$ Corresponding author. E-mail: yuan-deyi@163.com. development. The former is the prestage of female gametogenesis, comprising sporogenous cell differentiation and megaspore formation to the maturation of functional megaspores. The latter refers to the period from megaspore mitosis to cellularization and maturation of the female gametophytes (Reiser and Fischer, 1993). Any disruption in the process of ovule development can result in failure to form mature and fertile ovules. In general, the most prominent characteristics of sterile ovules are abnormalities or the absence of female germ units and failed development of the nucellus, with only the integument developing. This situation results in the lack of mature and functional synergid cells to guide the pollen tube into the embryo sac to release sperm cells, mature egg cells for sperm and egg fusion to produce fertilized eggs, which in turn develop into embryos, and mature central cells for fertilization to form a fertilized polar nucleus, which develops into the endosperm (Akhalkatsi et al., 1999; Casper and Wiens, 1981; Hu, 2005).

Camellia oleifera, originating in China, is a type of evergreen shrub or small tree in the Theaceae family. This important and unique woody tree species is a source of edible oil in southern China. Indeed, along with Cocos nucifera, Elaeis guineensis, and Olea europaea, C. oleifera is one of the world's four major woody oil plants (Zhuang, 2008). The contents of unsaturated fatty acids, oleic acids, and linoleic acids in $C$. oleifera can be as high as $90 \%, 75 \%$ to $83 \%$, and $7.4 \%$ to $13 \%$, respectively. The oil of $C$. oleifera, which has properties of softening blood vessels and lowering blood pressure and blood lipids, is known as "the longevity oil" and "the king of oil"; it 
is among the highest quality edible oils worldwide and is termed "oriental olive oil" (Gao et al., 2015a, 2015b; Lee and Yen, 2006). Because mature seeds are the major source for oil extraction, the cultivation and breeding objective of $C$. oleifera is to enhance the production of mature and full seeds. Because C. oleifera is unique to China, research on its embryology is commonly reported in studies by Chinese scholars. For example, Yuan et al. (2011) systematically studied the development of the female gametophyte of $C$. oleifera, noting onion-type embryo sac development in normal ovules. The megasporocyte undergoes meiotic division, giving rise to two dyad cells; however, only the megaspore at the chalazal end has a biological function, forming 7-cell, 8-nucleus embryo sacs. In addition, Gao et al. (2015b) carried out a detailed study of double fertilization in fertile embryo sacs of $C$. oleifera and reported premitotic gametogony double fertilization in this species. Cao (1965) performed karyotype analysis of $C$. oleifera endosperm development and found that endosperm cells are absorbed during embryo formation; thus, the mature seeds have no endosperm. The results of Gao et al. (2015c) and Liao et al. (2014) show that $C$. oleifera self-incompatibility is due to a prezygotic late-acting reproductive barrier. In general, mature seeds within fruit are important indicators of seed set under good cross-pollination conditions, which is often associated with mature fertile ovules in the ovary. To a certain extent, the number of fertile ovules in the ovary also reflects the number of mature and potential seeds that can develop into fruit. Conversely, abortive seeds cannot be harvested for economic gains.

C. oleifera is a self-incompatible plant, and fruit set is greatly reduced following self-pollination. Under cross-pollination, fruit set is reestablished; nevertheless, some dry and aborted seeds still form. Therefore, in this study, we explore the nascent structures of aborted seeds prefertilization and postfertilization to determine the physiological differences between fertile and aborted ovules. To this end, we compared fertile and abortive ovules, examining the developmental characteristics of aborted ovules and the number of sterile ovules in a mature ovary. Such a study of the developmental characteristics and occurrence of sterile ovules is of great significance to $C$. oleifera reproductive biology research and can provide basic information on the evolution and breeding of $C$. oleifera, as well as lay a theoretical basis for selecting cultivars with a higher seedsetting proportion.

\section{Materials and Methods}

\section{Materials}

Ten-year-old $C$. oleifera trees with good growth and normal flowering planted in Dongcheng Town, Wangcheng District, Changsha, Hunan Province (lat. $28^{\circ} 05^{\prime} \mathrm{N}$, long. $113^{\circ} 21^{\prime} \mathrm{E}$ ), were selected as the experimental material. All floral organs were selected during the blooming period and from the middle position of the trees. The site has an average annual precipitation of $1380 \mathrm{~mm}$, annual average temperature of $19.3^{\circ} \mathrm{C}$, annual accumulated temperature of $5463{ }^{\circ} \mathrm{C}$, annual average frost-free period of 276-291 d, and annual average sunshine of $1762 \mathrm{~h}$. It is a typical subtropical monsoon humid climate with sufficient light and heat. The soil is red soil which is typical of the hilly regions of central China that is developed mainly from quaternary red clay, with medium fertility and a $\mathrm{pH}$ value of $\approx 5.5$. Based on the method described by Gao et al. (2011) as well as the actual conditions of the experimental site, each plant was watered once per week (30 L) and given organic fertilizer once in winter $(10 \mathrm{~kg})$.

\section{Methods}

Cross-Pollination. During the full-blossom period, pollens were collected from the cultivar Xianglin XLC15. Then, artificial controlled pollination was performed for the cultivar Huashuo after castration ('Huashuo' $\times$ 'Xianglin XLC15'). The samples were capped with parchment paper bags and then tagged. Pistils not receiving pollination (on the blooming day) and those at $3,115,120,125,145,150,155,175,180$, and 185 DAP were collected.

Paraffin-Sectioning method. Samples were prepared according to Gao et al. (2015b). Pistils of $C$. oleifera were removed from the tree and fixed in a fixative solution $[95 \%$ ethanol:glacial acetic acid (v/v) 3:1] for $12 \mathrm{~h}$. After vacuum treatment, the material was transferred to $70 \%$ ethanol solution and stored in a refrigerator at $4{ }^{\circ} \mathrm{C}$. After proper trimming, the fixed materials were dehydrated in a gradient of different concentrations of ethanol $(30 \%, 50 \%, 70 \%, 80 \%, 90 \%$, and $100 \%)$, treated with xylene $(50 \%$ and $100 \%)$, and embedded in paraffin. The samples were sectioned using a microtome (RM2235; Leica, Heidelberg, Germany) to a thickness of $8 \mu \mathrm{m}$. The samples were adhered to slides, deparaffinized, rehydrated, treated with $4 \%$ ferric ammonium sulfate for $3 \mathrm{~h}$, and stained with $0.8 \%$ Ehrlich's hematoxylin for $25 \mathrm{~min}$; color separation was performed with $2.0 \%$ ferric ammonium sulfate for $20 \mathrm{~min}$. The samples were dehydrated and stained with $0.2 \%$ eosin, and permanent preparations were generated using Canada balsam. Images were captured under an optical microscope (BX-51; Olympus, Tokyo, Japan).

FLUORESCENCE ANALYSIS. This method is an observation approach based on paraffin sections and fluorescent staining. The sampled pistil materials were first prepared as paraffin sections at a thickness of $13 \mu \mathrm{m}$. The sections were deparaffinized and rehydrated without ferric ammonium sulfate treatment and were instead stained with $0.3 \%$ aniline blue solution for $2 \mathrm{~h}$. They were then placed on slides (without mounting), observed under a fluorescence microscope (BX-61, Olympus), and photographed.

SCANNING ELECTRON MICRoscopy (SEM). The material to be observed was carefully removed using forceps and a scalpel, fixed in $2.5 \%$ glutaraldehyde fixative (prepared by $0.1 \mathrm{~mol} \cdot \mathrm{L}^{-1}$ phosphate buffer) for $2 \mathrm{~h}$, and washed with phosphate buffer $\left(0.1 \mathrm{~mol} \cdot \mathrm{L}^{-1}\right)$. The samples were then fixed with $1 \%$ osmic acid fixative (prepared by $0.1 \mathrm{~mol} \cdot \mathrm{L}^{-1}$ phosphate buffer) for $2 \mathrm{~h}$, washed with phosphate buffer $\left(0.1 \mathrm{~mol} \cdot \mathrm{L}^{-1}\right)$, and dehydrated using an ethanol gradient $(30 \%, 50 \%, 70 \% 80 \%, 90 \%, 95 \%$, and $100 \%$ ). The samples were transferred to tert-butyl alcohol, and freeze-drying was carried out. Each sample was placed on the sample stage, placed in an ion-sputtering instrument for $20 \mathrm{~m}$ for gold plating and observed by SEM (JSM-6390; JEOL, Tokyo, Japan).

WHOLE-MOUNT STAINING/CLEARING COMBINED WITH CONFOCAL LASER SCANNING MICROSCOPY (CLSM). After collecting the pistils from the tree, the ovules were removed under an anatomical microscope and fixed immediately in fixative [95\% ethanol:glacial acetic acid (v/v) 3:1] for $6 \mathrm{~h}$. The material was then transferred to $70 \%$ ethanol solution and stored in a refrigerator at $4{ }^{\circ} \mathrm{C}$. The samples were stained with $1 \%$ eosin solution (prepared using $70 \%$ ethanol) for $1 \mathrm{~h}$, and the staining 
solution was removed. The samples were dehydrated using an ethanol gradient and treated with methyl salicylate transparent material for observation. Because of the large volume and the thickness of $C$. oleifera ovules, for observation, the ovules were placed on the concave surface of a concave glass slide. A few drops of methyl salicylate were placed around the ovule, and the cover slip was gently placed; the rim of the cover slip was sealed with small pieces of film. Another advantage of using the concave slide was that it is conducive to collect light and enhances the brightness for clear imaging by CLSM (SP8; Leica). The entire ovule was subjected to layered scanning, and images were obtained. A $63 \times$ oil immersion lens was used for observation, and the excitation wavelength was $543 \mathrm{~nm}$. The software (LAS AF; Leica) included with the device was used to carry out threedimensional reconstruction of multiple images.

\section{Statistical analysis of aborted ovules for different cultivars of $\boldsymbol{C}$. oleifera}

A total of 100 completely developed and matured ovaries that were not pollinated were randomly selected for each of the four cultivars (Huashuo, Huajin, Huaxin, and Xianglin XLC15) on the blooming day for sectioning. The numbers of normal and aborted ovules were counted. The average proportion of aborted ovules was calculated as the number of aborted ovules/ (number of normal ovules + number of aborted ovules). Data were analyzed using IBM SPSS Statistics (version 19.0; IBM Corp., Armonk, NY). Analysis of variance was performed with a confidence interval of 95\%. Pairwise comparisons were performed using the Duncan method if the $\mathrm{F}$ test showed a significant difference $(P<0.05)$.

\section{Results}

Developmental Characteristics OF OVULES IN DIFFERENT POSITIONS. In general, five to six ovules grew inside each $C$. oleifera ovary locule, which formed two rows symmetrically with two or three in each row; both rows are anatropous, and the ovule is connected to the placenta by a short funiculus. According to the observation results of the paraffin sections and fluorescence sections, the ovules attached on the bottom of the placenta normally had no complete embryo sac structure, which consisted only of inner and outer integuments, and the nucellus was rarely observed at this location. By contrast, the ovules located in the apical and intermediate positions along the placenta could be observed with a complete ovule structure consisting of the outer integument, inner integument, and embryo sac (Fig. 1A and B). Therefore, after cross-pollination, a large number of pollen tubes could be observed circling apical and intermediate ovules, whereas no pollen tubes were observed near the ovules at the bottom (Fig. 1B). SEM observation of fine differences in the overall morphology of ovules at different positions along the placenta revealed that plumper, smoother, and fuller ovules (Fig. 1C) at the apical and intermediate positions were packed
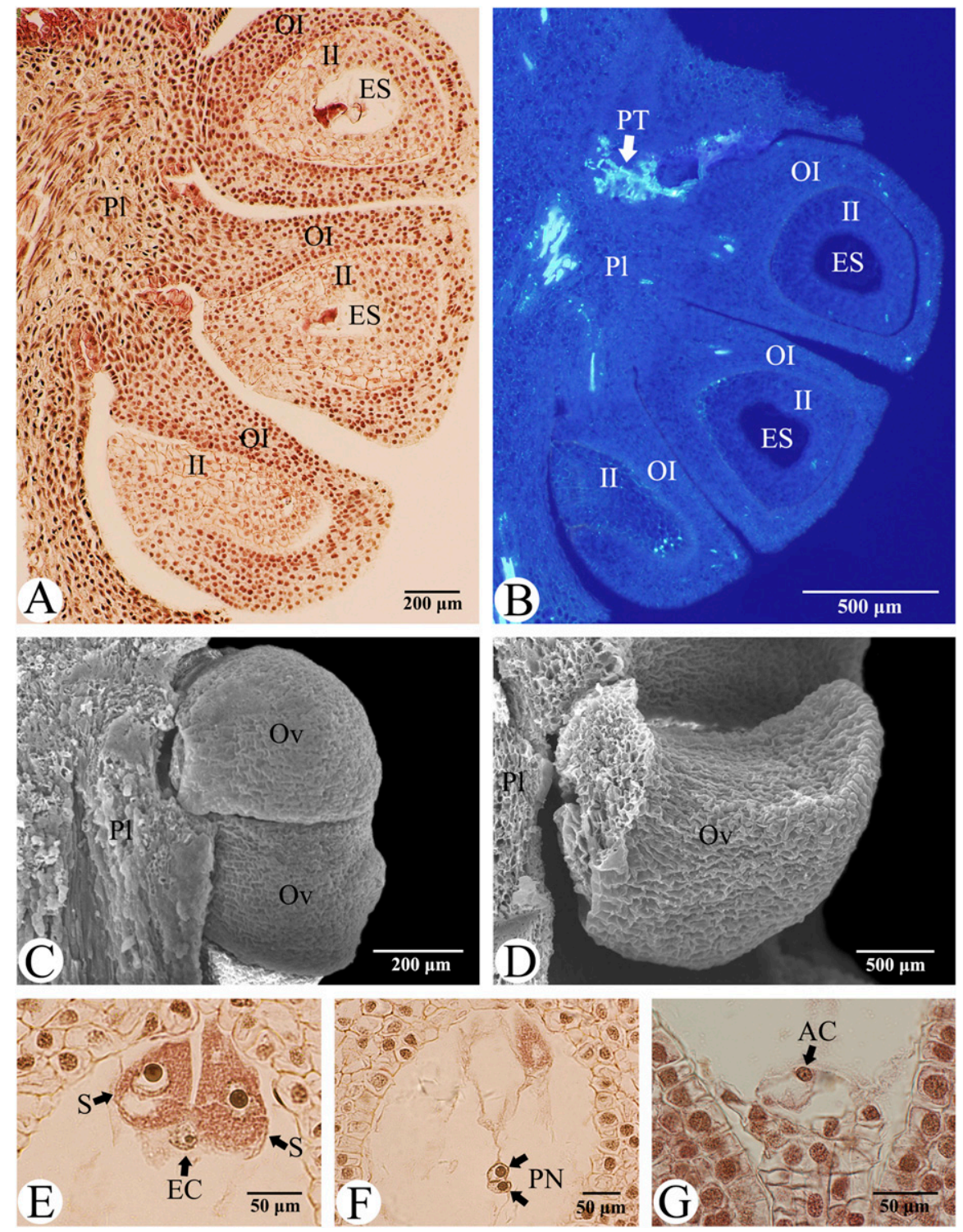

Fig. 1. Position and characteristics of ovules (Ov) in the ovary of Camellia oleifera were observed. (A) A row of anatropous ovules in the ovary are attached to the placenta via a short funiculus on the blooming day. (B) According to the section fluorescence observation of a row of ovules at $3 \mathrm{~d}$ after pollination, ovules in the ovary at the apical and intermediate positions of the placenta (Pl) are surrounded by pollen tubes (PTs), whereas ovules at the bottom position are not. (C) Full and round ovules in the ovary are at the apical and intermediate positions at blooming day according to scanning electron microscopy (SEM). (D) Angular, dry ovules at the bottom shows signs of contraction at blooming day according to SEM. (E) Synergid (S) and egg cells (EC) in the ovules are located at the apical and intermediate positions of the placenta at blooming day. (F) Polar nucleus (PN) is observed in ovules at the apical and intermediate positions on the blooming day. (G) Antipodal cells (AC) are observed in ovules at the apical and intermediate positions at blooming day; ES = embryo sac, II = inner integument, OI = outer integument. 
tightly next to each other, while ovules at the bottom position appeared angular and were relatively thin and dry, with signs reminiscent of desiccation (Fig. 1D). Based on the sections of the apical and intermediate positions of ovules, a complete structure of female reproductive organs was found in the embryo sac: The two synergid cells appeared pear shaped, and the chalazal end of the ovule contained large vacuoles; the egg cells were closely connected to the synergid cells (Fig. 1E), showing a "pyramid" structure; the two polar nuclei were located below the egg apparatus (including an egg cell and two synergids) (Fig. 1F); and the antipodal cells were not noticeable, with a tendency toward degeneration (Fig. 1G). By contrast, ovules located at the bottom were rarely observed with the complete embryo sac structure.

DeVElOPMENTAL TYPE AND CHARACTERISTICS OF ABORTED OVULES. In this study, whole-mount staining/clearing combined with CLSM and the paraffin sectioning method combined with optical microscopy were used to observe the aborted ovules at the bottom of the ovary. The developmental types and characteristics of these aborted ovules can be grouped into four categories. In the first category, the egg apparatus structure was barely visible in the nucellus of the aborted ovules, but without a cell outline, resembling degenerated cells with a narrow embryo sac cavity (Fig. 2A-C). In the second category, the egg apparatus structure was absent in the nucellus of aborted ovules, consisting mainly of flocculent tissue and a narrow embryo sac cavity (Fig. 2D-F). In the third category, no tissue was observed in the nucellus of aborted ovules, which were filled with integument cells with only a narrow crevice (Fig. 2G-I). For the fourth category of the aborted ovules, some showed developmental abnormalities, compared with a rather long inner integument and micropyle channel part in a normal ovule (Fig. 2J). These ovules had a short micropyle channel formed by the inner integument, and immature synergid cells were directly located at the micropylar opening. No pollen tubes were found to enter the underdeveloped egg apparatuses (Fig. 2K). In comparison with the normal embryo

A

D sac, the synergid cells, egg cells, and polar nucleus were all stunted, with indications of degeneration. A three-dimensional reconstruction of the incompletely developed egg apparatus of
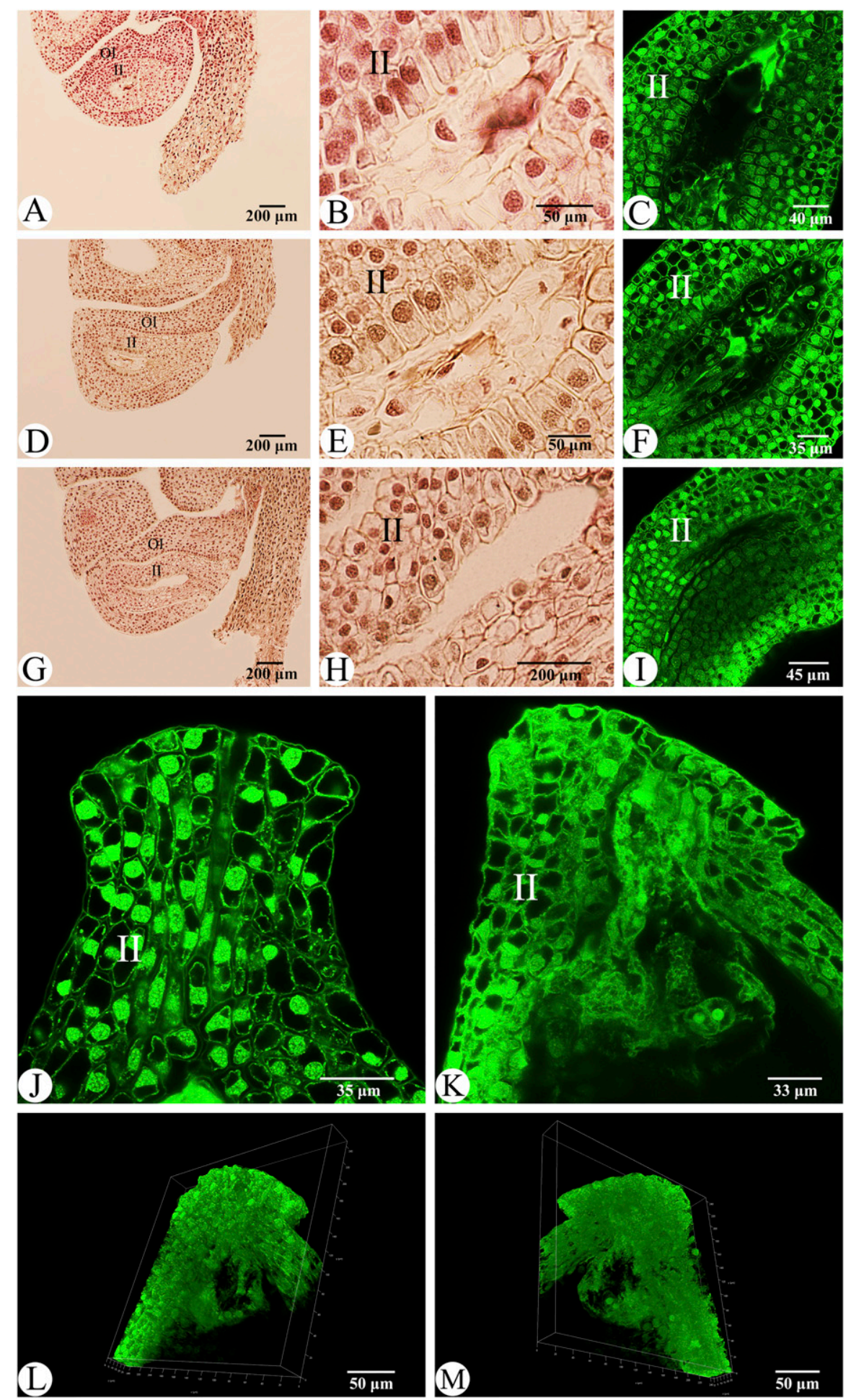

Fig. 2. Developmental characteristics of an aborted ovule of Camellia oleifera were observed using laser confocal microscopy. (A-C) The first category of aborted ovules, in which the egg apparatus structure is distinctly visible in the nucellus, though with no cell contour. (D-F) The second category, in which the egg apparatus structure is completely absent, consisting mainly of flocculent tissue. (G-I) The third category, with no tissue found in the nucellus, comprising integument cells with only a narrow crevice. (J) The inner integument. (H) Micropyle channel of normal ovules under confocal microscopy. (K) The fourth category, in which the inner integument, embryo sac, and micropyle channel are absent and the egg apparatus is not fully developed. (L and M) Threedimensional reconstruction imaging of abnormal ovules from different angles; OI = outer integument. 
this type of abnormal ovules was constructed using CLSM. The image was rotated and then observed from different angles (Fig. 2L and M). Obvious differences between these abnormal ovules and normally developed ovules could also be observed: direct growth of synergid cells at the micropylar opening, an incomplete cell membrane, and a thin cytoplasm. For these aborted ovules, the integument cells also began to degenerate in the late stage of development, and cell contours became fuzzy.

LATE-STAGE DEVELOPMENT OF FERTILE AND ABORTED OVULES. Following pollination in C. oleifera, the zygote entered a dormant state until it begins to split at 150 DAP. At $\approx 120$ DAP, the ovary began to expand, and its size began to increase. When the zygote splits, the fertile and aborted ovules exhibited noticeable size differences. At this point, the fertile ovule had increased significantly in volume and tended to be white in color. Conversely, the aborted ovule had increased in size only slightly and was a deep-yellow color. Thus, a remarkable contrast between the two types was observed (Fig. 3A). At 120 DAP, a row of ovules was sectioned and observed, and it was found that the inner and outer integuments of both fertile and aborted ovules had obviously thickened, with the gap between the integuments of the fertile ovules being larger than that between the integuments of the aborted ovules. In addition, at this point, the embryo sac cavity of the fertile ovules was increased compared with that before fertilization, whereas the nucellus of the aborted ovules was blurred and squeezed together by the inner integument (Fig. 3B and C). At 180 DAP, the size differences between the fertile and abortive ovules were clearly visible (Fig. 3D). After transectioning, the endosperm of the fertile ovule was gel-like, and the inner and outer integuments were clearly visible. However, the integument of the aborted ovule was blurred, and the nucellus was filled with integument cells (Fig. 3E); furthermore, the aborted ovules stopped developing at this moment. Both the fertile and aborted ovules at 180 DAP were separated off and then observed under SEM. Their differences in morphology were much more noticeable than those on the blooming day. The fertile ovules already exhibited the rudimentary form of the mature seeds with full morphology, whereas the aborted ovules were markedly withered and sunken, showing a tendency toward degeneration (Fig. 3F and G), and the aborted ovule had already ceased to develop. The aborted ovules at this time were sectioned and observed using fluorescence microscopy. In the abnormal ovules of the fourth category, the initially formed "egg apparatus" within the embryo sac had entirely degenerated, and the inner integument was wrinkled; the cells
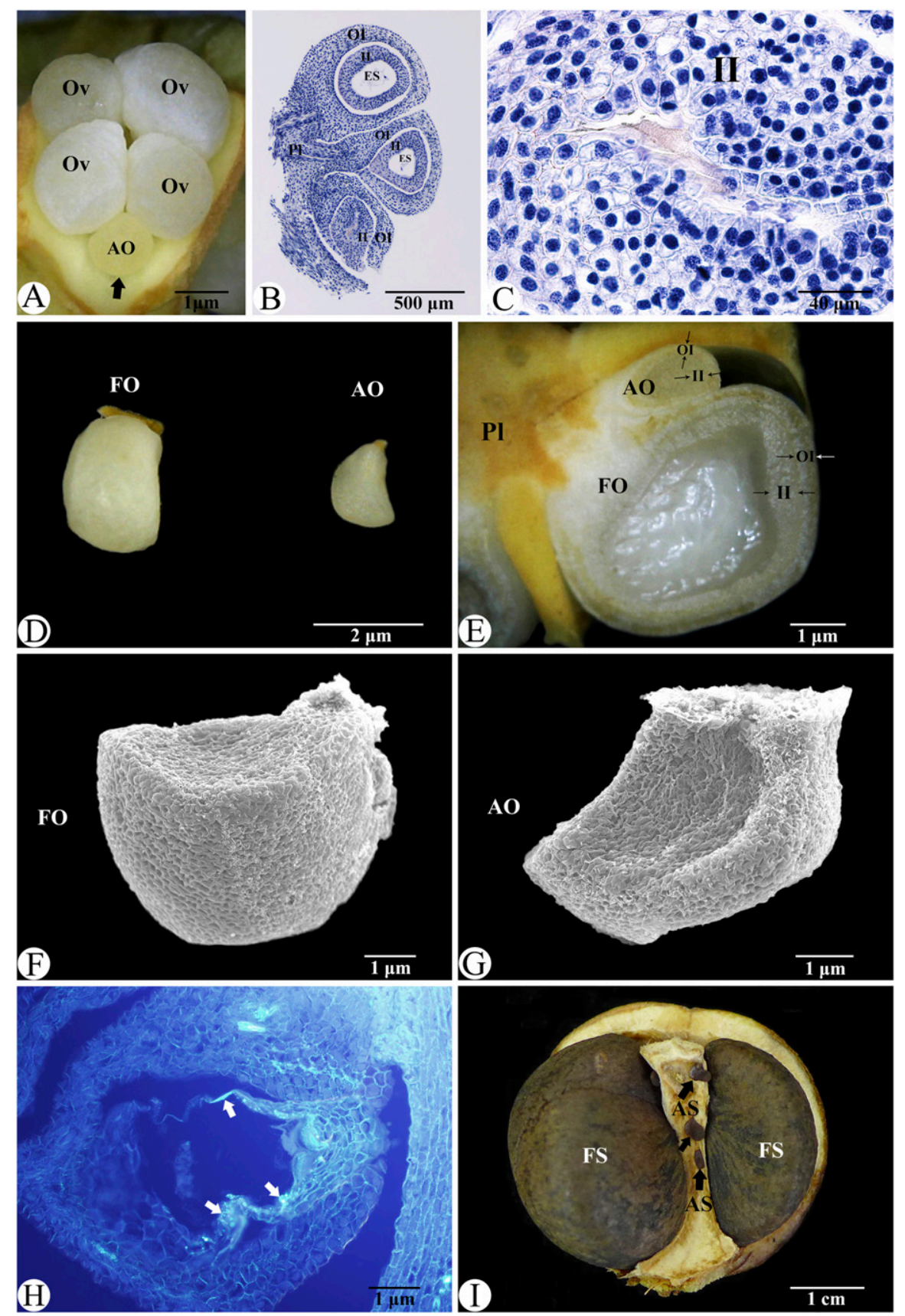

Fig. 3. Late-stage development of fertile ovules (FOs) and aborted ovules [AOs (shown by black arrows)] of Camellia oleifera were observed. (A) At $120 \mathrm{~d}$ after pollination (DAP), fertile and aborted ovules are identifiable in an ovary. (B) Longitudinal section of a row of ovules $(\mathrm{Ov})$ at $120 \mathrm{DAP}$, where the distinction between the fertile and aborted ovules is obvious. (C) Magnification of a blurred and disappeared nucellus is observed in an aborted ovule shown in B. (D) Remarkable differences in size and shape are observed between fertile fertilized ovules (left) and aborted ovules (right). (E) At 180 DAP, an expanded fertilized ovule and aborted ovule. (F) A fertile ovule during development at 180 DAP. (G). An aborted ovule at 180 DAP. (H) Late development of an aborted ovule at 180 DAP (the bright spot in the ovule shown by the white arrow). (I) A fertile seed (FS) and an aborted seed (AS) in a mature fruit, in which the aborted seed (shown by black arrows) grows dry and browned on the placenta $(\mathrm{Pl})$; ES = embryo sac, II = inner integument, OI = outer integument, Ov = ovule. 
had disintegrated and were enclosed only by the outer integument. In addition, a fluorescent bright spot within the inner integument was observed (Fig. 3H). Following pollination, fertile ovules formed fully developed seeds, whereas aborted ovules formed dry aborted seeds attached to the placenta (Fig. 3I). The proportions of aborted ovules for four major $C$. oleifera cultivars under cross-pollination conditions were also calculated in this study, and the results were as follows (Table 1): $13.3 \%$ to $20.2 \%$ for 'Huashuo', $15.6 \%$ to $33.3 \%$ for 'Huajin', $10.2 \%$ to $20.5 \%$ for 'Huaxin', and $20.8 \%$ to $30.7 \%$ for 'Xianglin XLC15'. Although these aborted ovules increase in size during early ovary development to some extent, they would gradually become dry and brown in late development, eventually forming aborted seeds in mature fruit.

\section{Discussion}

As an important reproductive organ of angiosperms, the ovule has received consistent attention from botanical researchers. Particularly, studies on food, breeding, and grafting related to plant seeds will be of great significance for human production practice. The ability to obtain mature and fully fertile seeds is important for the production of $C$. oleifera. First, these seeds can be used for extracting edible oil, oil tea, and camellia seed oil. Second, the fertile seeds can be used to cultivate grafting plants after embryo roots grow out. In addition, the matured seeds obtained from purposeful hybridization can be used for backcrossing and testcrossing. Therefore, the ability to obtain more mature and full seeds is the target of $C$. oleifera cultivation and breeding, no matter the angle of production or research. The maturation of the seeds is closely associated with the fertile ovules in the ovary, and the number of fertile ovules in the ovary reflects the number of mature seeds in fruit to a certain degree. The abortion of seeds will inevitably influence economic profit and seed breeding efficiency because aborted seeds cannot be used at harvest.

In the mature ovary of $C$. oleifera, we observed that ovules grow on the placenta in the ovary and that development may vary according to position. In general, five to six ovules are present in an ovary, with two to three ovules in each row. The ovules growing on the placenta (above the stylar end) are well developed and those located at the bottom of the placenta (at the pedicel end) are often stunted and aborted. Gao et al. (2015b) reported double fertilization and the position of the ovule in normal fertile ovules of $C$. oleifera but did not mention differences in ovule development at different positions. Combined with the characteristics of C. oleifera (Deng et al., 2010; Gao et al., 2015a), the reason for the developmental differences in ovules at different positions is presumably due to the reproductive characteristics and the distribution of nutrients. Thus, an active abortion mechanism has existed during long-term evolution. Because $C$. oleifera is a self-incompatible plant that is

Table 1. Proportion of aborted ovules in ovaries of four major cultivars of Camellia oleifera.

\begin{tabular}{lcc}
\hline Cultivar & Proportion of aborted ovules (\%) & Avg proportion (\%) \\
\hline Huashuo & $13.3-20.2$ & $16.75 \mathrm{a}^{\mathrm{z}}$ \\
Huajin & $15.6-33.3$ & $24.45 \mathrm{~b}$ \\
Huaxin & $10.2-20.5$ & $15.35 \mathrm{a}$ \\
Xianglin XLC15 & $20.8-30.7$ & $25.75 \mathrm{~b}$
\end{tabular}

${ }^{\mathrm{z}}$ Average proportions were compared between groups using the Duncan method. Different letters indicate significant differences at $P<0.05$. insect pollinated, the fruit set percentage of self-pollination is very low. C. oleifera blooms in winter when the number of pollinating insects is small and when the type of pollinators is also limited. As a result, there is insufficient heterogeneous pollen for normal pollination. To ensure seed formation for future multiplication, it is necessary that tightly packed and well-developed ovules receive a limited amount of heterogeneous pollen tubes for fertilization. Accordingly, the apical and intermediate ovules adjacent to the stylus have priority, and consequently, their development is better than that of ovules located at the bottom (Deng et al., 2010; Liao et al., 2014). There are also reports in other plants on developmental differences in ovules due to differences in position. In Cucumis sativus (Jing et al., 2000), the quality of seeds located at the stylar end and the peduncular end was lower than that of seeds at other positions. The same conclusion was reached for Phaseolus coccineus (Rocha and Stephenson, 1990). However, there are few reports on the cause of this difference. In particular, in years with a dry climate and little rain, coupled with the lack of fertilizer in the natural environment, priority in terms of nutrients supply is given to the development of ovules at the apical and intermediate positions. Nonetheless, further research is needed on the stage of development during which abortion occurs for the lower portion of ovules and how nutrients are metabolized and transferred.

Based on observation of the sections and confocal microscopy, aborted ovules were present on the day of flowering rather than being formed during the process of development after fertilization. Overall, aborted ovules can be divided into four categories, each with different characteristics. Although the integument of aborted ovules still increases in size, as does that of normal ovules, a large hollow cavity is not formed in the center. There is little space for these sterile ovules to develop, especially in the late stage when the embryo sac is solid. In general, this appears to be the result of failure of the nucellus to develop normally and its abortion before maturation of the embryo sac. Cao (1965) provided a brief description of aborted seeds in the ovary of $C$. oleifera but did not report in detail on the causes and processes of abortion. Other studies on the embryology of $C$. oleifera have focused on the formation and development of normal female and male reproductive organs, but there is no detailed report on the formation and late development of sterile ovules. According to observations of the number of aborted ovules among four cultivars of $C$. oleifera, we found that abortion proportions range from $10.2 \%$ to $33.3 \%$ (Table 1 ). Similarly, aborted ovules before fertilization have been found in several species. In Medicago sativa (Wang et al., 2011), there were significant differences in the abortion proportions of ovules of different cultivars, with the highest reaching 49.07\%. An average abortion percentage of 39\% was reported for Epilobium obcordatum (Seavey and Carter, 1996), and abortion was observed in $10.5 \%$ of Lotus corniculatus ovules (Rim et al., 1990). To a certain extent, these aborted ovules reduced the number of mature seeds. The sterile ovules of $C$. oleifera continue to increase in size with development of the fertilized ovary. At 120 DAP, the size of fertile and aborted ovules differs, and the aborted ovule ceases to grow by 180 DAP, becoming gradually withered and brown and ultimately constituting the abortive seeds of the mature fruit. These ovules also have complete outer and inner integuments, a tapetum, and 
a nucellus. However, the nucellus, which disappears before the embryo sac matures but cannot form the embryo sac. From an embryological point of view, the plant ovule undergoes a long process from the beginning of growth and development to the mature seed. Abortion can occur at any stage, from the generation of megaspore mother cells to megaspore mother cell meiosis through formation of the female gametophyte and even late in fertilized ovule development ( $\mathrm{Hu}, 2005)$. There are many possible causes of abortion. For example, after the formation of megaspore mother cells, they cannot undergo meiosis normally, rendering them unable to produce functional megaspores; this is the most common phenomenon. In addition, in individual aborted ovules, although the megaspore mother cells within the nucellus cells are visible, cell vacuolization or apoptosis before meiosis can occur, for example, in L. corniculatus (Rim et al., 1990). With further development of the functional megaspores and formation of functional megaspores after the normal completion of meiosis, the mitotic process is blocked, resulting in failure to produce a mature seven-cell, eight-nucleus embryo sac. Lillecrapp et al. (1999) reported this in apricot. Because ovule abortion occurs before the maturation of the ovary, no pollen tubes will extend into the aborted ovule during their growth in the ovary. Ovule abortion also occurs during seed formation, that is, a mature embryo sac in ovules that can undergo normal fertilization and pollination is present. However, ovules with abnormal development later fail to generate normal seeds. Indeed, ovules within many mutations have very low fertility because of their development abnormalities. As with male sterility and the self-incompatibility of higher plants, the causes of plant ovule abortion are complex. During reproduction, abnormal metabolism, changes in nutrients and hormone levels, and changes in enzyme activity within the plant can all lead to ovule abortion. Changes in these molecules are closely related to gene regulation. In addition to genetic factors, environmental factors also trigger ovule abortion. The development of plant reproductive organs requires a suitable ecological environment, and changes in external factors may also affect the differentiation and development of female organs, resulting in stagnation and abortion of ovule development. In the future, determining whether the changes in environment factors have an effect on the abortion of $C$. oleifera will be an important research direction. Specifically, whether the lack of timely irrigation in dry and hot weather in summer will have significant influence on the flower bud differentiation and ovule development of $C$. oleifera in the same year in the $C$. oleifera growth areas of China is worth future investigation. Furthermore, the changes that occur to the nutrient substances, hormones, and enzymic activity in the fertile and aborted ovules of $C$. oleifera in dry/high temperature environment are another focus of further studies. In addition, whether the changes in the factors of the environment in which $C$. oleifera tress grow will result in differences in the abortion proportion of C. oleifera remain to be explored.

\section{Literature Cited}

Akhalkatsi, M., M. Pfauth, and C.L. Calvin. 1999. Structural aspects of ovule and seed development and nonrandom abortion in Melilotus officinalis (Fabaceae). Protoplasma 208:211-223.
Cao, H.J. 1965. Embryological observation on Camellia oleifera. Acta Bot. Sin. 13:44-53.

Casper, B.B. and D. Wiens. 1981. Fixed rates of random ovule abortion in Cryptantha flava (Boraginaceae) and its possible relation to seed dispersal. Ecology 62:866-869.

Deng, Y.Y., X.L. Yu, and Y.B. Luo. 2010. The role of native bees on the reproductive success of Camellia oleifera in Hunan Province, Central South China. Acta Ecol. Sin. 30:4427-4436.

Endress, P.K. 2011. Angiosperm ovules: Diversity, development, evolution. Ann. Bot. (Lond.) 107:1465-1489.

Gao, C., D.Y. Yuan, B.F. Wang, Y. Yang, D.M. Liu, and Z.Q. Han. 2015a. A cytological study of anther and pollen development in Camellia oleifera. Genet. Mol. Res. 14:8755-8765.

Gao, C., D.Y. Yuan, Y. Yang, B.F. Wang, D.M. Liu, and F. Zou. 2015b. Pollen tube growth and double fertilization in Camellia oleifera. J. Amer. Soc. Hort. Sci. 140:12-18.

Gao, C., D.Y. Yuan, Y. Yang, B.F. Wang, D.M. Liu, F. Zou, and X.F. Tan. 2015c. Anatomical characteristics of self-incompatibility in Camellia oleifera. Scientia Silvae Sinicae 2:60-68.

Gao, C., D.Y. Yuan, J. Yuan, Y.Q. Qiu, and G.J. Gan. 2011. Influences of sod culture on the physical and chemical characteristics of soil in hilly Pyrus pyrifolia orchard of eastern Hunan. Hubei Agr. Sci. 8:1593-1595.

Hu, S.Y. 2005. Reproductive biology of angiosperms. China Higher Educ. Press, Beijing, China.

Jing, H.C., J.H.W. Bergervoet, H. Jalink, M. Klooster, S.L. Du, R.J. Bino, H.W.M. Hilhorst, and S.P.C. Groot. 2000. Cucumber (Cucumis sativus L.) seed performance as influenced by ovary and ovule position. Seed Sci. Res. 10:435-445.

Lee, C.P. and G.C. Yen. 2006. Antioxidant activity and bioactive compounds of tea seed (Camellia oleifera Abel.) oil. J. Agr. Food Chem. 54:779-784.

Liao, T., D.Y. Yuan, F. Zou, C. Gao, Y. Yang, L. Zhang, and X.F. Tan. 2014. Self-sterility in Camellia oleifera may be due to the prezygotic late-acting self-incompatibility. PLoS One 9:e99639.

Lillecrapp, A.M., M.A. Wallwork, and M. Sedgley. 1999. Female and male sterility cause low fruit set in a clone of the 'Trevatt' variety of apricot (Prunus armeniaca). Sci. Hort. 82:255-263.

Reiser, L. and R.L. Fischer. 1993. The ovule and the embryo sac. Plant Cell 5:1291-1301.

Rim, Y.W., P.R. Beuselinck, R.L. McGraw, and D.A. Somers. 1990. Megagametophyte development in Lotus corniculatus, L. conimbricensis, and their protoplast fusion hybrid. Amer. J. Bot. 77:1084-1094.

Rocha, O.J. and A.G. Stephenson. 1990. Effect of ovule position on seed production, seed weight, and progeny performance in Phaseolus coccineus L. (Leguminosae). Amer. J. Bot. 75:1320-1329.

Seavey, S.R. and S.K. Carter. 1996. Ovule fates in Epilobium obcordatum (Onagraceae). Amer. J. Bot. 83:316-325.

Shi, D.Q. and W.C. Yang. 2011. Ovule development in Arabidopsis: Progress and challenge. Curr. Opin. Plant Biol. 14:74-80.

Skinner, D.J., T.A. Hill, and C.S. Gasser. 2004. Regulation of ovule development. Plant Cell 16:S32-S45.

Wang, X., X. Li, J.W. Zhang, G.H. Feng, S.Z. Zhang, L.C. Huang, R.Y. Zhuo, and L. Jin. 2011. Characterization of nine alfalfa varieties for differences in ovule numbers and ovule sterility. Austral. J. Crop Sci. 5:447-452.

Yuan, D.Y., F. Zou, X.F. Tan, C.Y. He, J. Yuan, and X.M. Fan. 2011. Flower bud differentiation and development of male and female gametophytes in Camellia oleifera. J. Central South Univ. For. Technol. 31:65-70.

Zhuang, R.L. 2008. Camellia oleifera in China. China For. Press, Beijing, China. 